Cinémas

Revue d'études cinématographiques

Journal of Film Studies

\title{
Pouvoir intime : récit scénaristique, récit filmique
}

\section{Denis Desaulniers}

Volume 2, numéro 1, automne 1991

Le Scénario

URI : https://id.erudit.org/iderudit/1001052ar

DOI : https://doi.org/10.7202/1001052ar

Aller au sommaire du numéro

Éditeur(s)

Cinémas

ISSN

1181-6945 (imprimé)

1705-6500 (numérique)

Découvrir la revue

Citer cet article

Desaulniers, D. (1991). Pouvoir intime : récit scénaristique, récit filmique.

Cinémas, 2(1), 66-91. https://doi.org/10.7202/1001052ar

\section{Résumé de l'article}

Pour que le film existe, il faut qu'il y ait « transcodage », c'est-à-dire passage d'un mode de signification à un autre. On pourrait dire que le passage du récit scénaristique au récit cinématographique se présente comme une véritable mutation où, d'un être essentiellement de mode nominatif, naîtra une créature hybride qui procède à la fois du mode nominatif et du mode représentatif. C'est ce processus de mutation qui est ici abordé, en esquissant une typologie des transformations que peut subir le récit scénaristique lors de sa réécriture sous la forme d'un récit cinématographique, puis au moyen de l'analyse comparative du récit scénaristique et du récit cinématographique du film Pouvoir intime d'Yves Simoneau (scénarisé par Pierre Curzi et Yves Simoneau). 


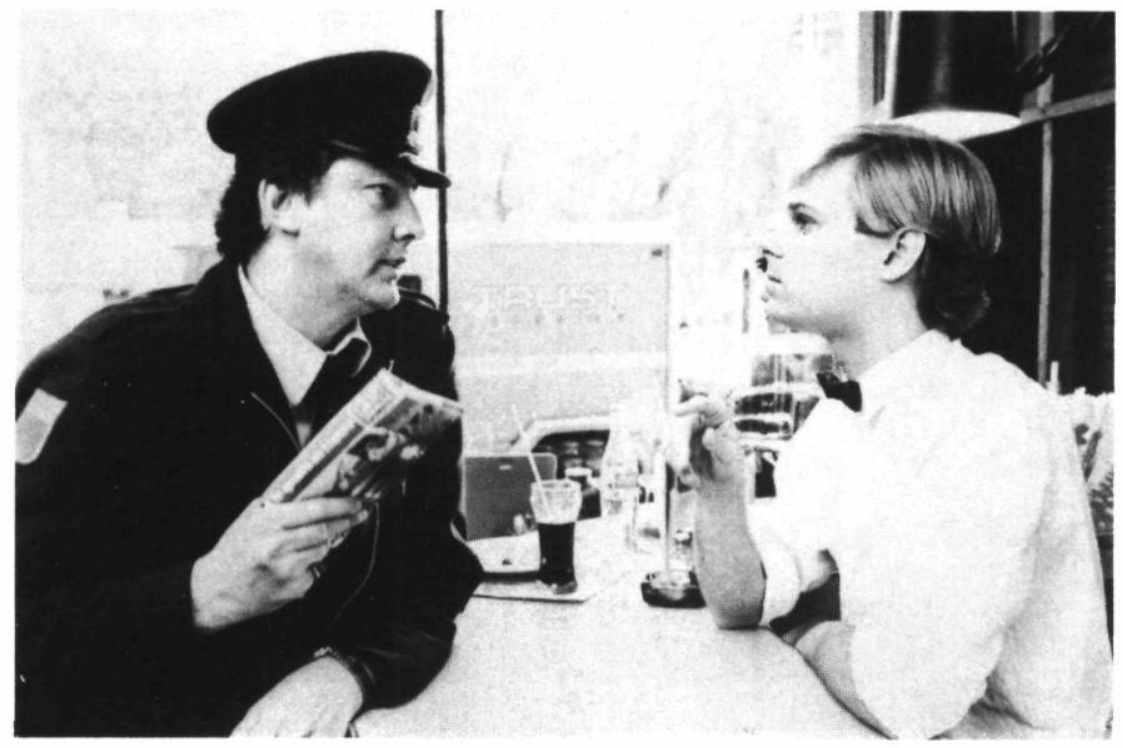

Pouvoir intime de Yves Simoneau (1986)

Coll. Cinémathèque québécoise 


\title{
Pouvoir intime: récit scénaristique, récit filmique
}

\section{Denis Desaulniers}

\begin{abstract}
RÉSUMÉ
Pour que le film existe, il faut qu'il y ait «transcodage», c'est-à-dire passage d'un mode de signification à un autre. On pourrait dire que le passage du récit scénaristique au récit cinématographique se présente comme une véritable mutation où, d'un être essentiellement de mode nominatif, naîtra une créature hybride qui procède à la fois du mode nominatif et du mode représentatif.

C'est ce processus de mutation qui est ici abordé, en esquissant une typologie des transformations que peut subir le récit scénaristique lors de sa réécriture sous la forme d'un récit cinématographique, puis au moyen de l'analyse comparative du récit scénaristique et du récit cinématographique du film Pouvoir intime d'Yves Simoneau (scénarisé par Pierre Curzi et Yves Simoneau).
\end{abstract}

\footnotetext{
ABSTRACT

In order for a film to exist there must be a "transcoding", a passage from one signifying mode to another. One might say that the movement from screenplay version to cinematic version can be seen as a real modification in which something whose mode is essentially nominative gives birth to a hybrid creature in which the nominative mode is combined with the representational mode.

This process of modification is examined here first by outlining a typology of the transformations that a screenplay version may undergo when it is recoded into a cinematic version, and then by making a comparative analysis of the screenplay and the cinematic versions of the film Pouvoir intime by Yves Simoneau (screenplay by Pierre Curzi and Yves Simoneau).
} 
Que le cinéma raconte des histoires, qu'il nous mette en présence d'un récit, nul ne conteste ce fait facilement vérifiable par tout(e) spectateur(rice) de cinéma. Cependant avant d'apparaître à l'écran, le récit d'un film est, pour bon nombre d'œuvres, d'abord narré sous la forme d'un scénario: c'est un récit scriptural singulier appelé récit scénaristique.

Ce récit est transmis par l'intermédiaire de l'écriture, à la différence du récit filmique qui, lui, s'actualise à travers les cinq matières de l'expression déjà définies par Christian Metz: les dialogues, les bruits, la musique, les mentions écrites et l'image. Il faut donc, pour que le film existe, qu'il y ait «transcodage», «transsémiotisation», c'est-à-dire qu'il y ait passage d'un mode de signification à un autre. L'on pourrait dire que cette transformation du récit scénaristique en récit filmique se présente comme une véritable mutation où, d'un être essentiellement de mode nominatif, naîtra une créature hybride qui procède des modes nominatif et représentatif. Parfois ce passage, cette «renaissance», se fait sans douleur. D'autres fois, le mutant est méconnaissable: «La plupart du temps, les metteurs en scène m'ont démoli mes scénarios», proteste Edward Chadorov ${ }^{1}$.

Cette mutation du récit scénaristique, nous tenterons de la cerner à travers les modifications qu'elle subit lors de sa «réécriture» en récit filmique. À cette fin, nous avons choisi pour illustrer notre propos un film d'Yves Simoneau que le réalisateur a lui-même scénarisé avec Pierre Curzi: Pouvoir intime $^{2}$. L'œuvre de Simoneau se prête bien à cet exercice puisque le style d'écriture du récit scénaristique laisse au réalisateur une confortable marge de manœuvre pour son «transfert» visuel.

Nous considérerons comme écart au récit scénaristique l'absence ou la présence modifiée dans le récit porté à l'écran d'une indication qui a d'abord été inscrite dans le texte du scénario. En ce qui concerne l'organisation des scènes, nous considérerons également comme écart au récit scénaristique tout ajout, retrait ou déplacement, total ou partiel, de scènes. Évidemment ces points de comparaison seront plus ou moins nombreux selon le style d'écriture du scénariste.

Par rapport à celui du scénario, le récit du film de Simoneau présente des modifications de deux ordres. Ces changements affectent soit la macrostructure, soit la microstructure du récit.

L'on désigne par «macrostructure» l'organisation globale du récit. Ainsi le déplacement d'une scène, par exemple, affectera la position de chacune des autres scènes dans la chaîne événementielle. Une modification macrostructurelle peut aussi se présenter sous la forme d'un changement qui devient par la suite ré- 
current à travers tout le récit. Si un personnage est décrit dans le scénario comme ayant une chevelure blonde et que lors de son apparition dans l'univers filmographique il a les cheveux noirs, même si elle n'affecte pas en soi l'ensemble de la structure narrative comme telle, cette transformation sera récurrente lors de chacune des apparitions du personnage à l'écran. C'est en ce sens que l'on qualifie cette modification de macrostructurelle puisqu'elle navigue à travers tout le récit filmique, émergeant à la surface lorsque nécessaire au développement de l'action.

La «microstructure», quant à elle, est assimilable à la scène ${ }^{3}$. Les modifications de cette catégorie seront donc d'ordre «intraséquentiel»; c'est dire que cette altération du récit se retrouvera à l'intérieur de la scène sans que, néanmoins, ce changement n'ait de répercussions majeures sur la totalité du récit filmique. Autrement dit, une modification microstructurelle n'affecte en rien l'organisation «interséquentielle» du récit.

\section{Modifications d'ordre macrostructurel}

Par opposition au scénario, le récit filmique de Pouvoir intime présente trois types de modification qui affectent son organisation macrostructurelle.

Le premier de ces changements touche la caractérisation des personnages ${ }^{4}$. D'abord le nom: il s'agit de l'individu qui s'enferme à l'intérieur du camion blindé et refuse d'en sortir. Le récit scénaristique nous le fait connaître sous le nom de JeanBaptiste. À l'écran, il s'appellera Martial.

L'incarnation écranique du personnage de Martial nous permet de constater un autre type de modification possible de la caractérisation des personnages. Ce changement touche plus particulièrement l'aspect physique de ces derniers. À la section Description des personnages du texte scénaristique, on peut lire au paragraphe relatif à Jean-Baptiste (Martial dans le film): «40 ans, cheveux raides et teints en blond, moustache» (Curzi et Simoneau, p. 2). À l'écran, les cheveux raides et blonds disparaissent pour faire place à des cheveux foncés et légèrement bouclés. La moustache a elle aussi disparu.

La blondeur des cheveux sera prise en charge dans le récit filmique par Janvier, l'amoureux de Martial (Jean-Baptiste dans le scénario). La moustache, quant à elle, devient à l'écran un attribut de l'un des complices: Gildor. Notons qu'il n'est fait aucune mention dans le scénario à la rubrique Description des personnages de la couleur des cheveux de Janvier et du port ou non de la moustache par Gildor.

La blondeur caractérise un autre personnage du récit scénaristique. Cette fois il s'agit de H.B., le fonctionnaire impliqué 
dans le vol: «30 ans, blond, yeux clairs, l'air presque fragile» (p. 3 ). Cette blondeur tourne au foncé dans la diégèse filmographique.

À la constitution physique des personnages, on pourrait rajouter dans le processus de caractérisation leur condition physique, leur santé. C'est en tout cas ce que font Curzi et Simoneau. Cette information n'est importante que dans la mesure où elle a une utilité dramatique. Ce sera le troisième type de transformation repérable dans cette œuvre en relation avec la caractérisation des personnages. Thomas, le complice de la Brink's (c'est lui qui doit être dans le camion blindé au moment du vol), est dans le récit scénaristique un personnage «nerveux» (p. 3) et asthmatique. À la scène $27 \mathrm{~A}$, alors qu'il sait que le vol se produira la journée même, Thomas se sent mal.

JEAN-BAPTISTE. - J'te rapporte ton lunch... t'a pas l'air à filer, qu'est-ce qu'y a?

THOMAS, blême, s'empresse de répondre. - Y a rien! C'est mon asthme... (p. 61).

Plus loin, à la scène 30 , Jean-Baptiste surprend aux toilettes des hommes Thomas qui a, malgré les consignes, laissé sans surveillance le camion blindé.

JEAN-BAPTISTE, surpris. — Qu'est-ce que tu fais là?!!

THOMAS, péniblement. - Le déjeuner (sic) passe pas... (p. $65)$.

Dans l'univers filmographique, ce détail de la mauvaise digestion de Thomas semble s'être imposé. Pour justifier son malaise, sa blancheur, Thomas ne fait plus référence à son asthme; il répond plutôt à Martial en ces termes: «Y a rien! C'est mon estomac.» Ce changement dans la caractérisation de Thomas nous semble renforcer davantage l'aspect «nerveux» du personnage. $\mathrm{Ni}$ dans le récit scénaristique, ni dans celui du film à l'écran, les motivations de la participation de Thomas à ce vol ne sont précisées. À l'écran son comportement, plus près de l'anxiété que de la simple nervosité, laisse croire qu'il en est à son "premier coup» ou, à tout le moins, qu'il n'a pas encore acquis le calme du professionnel. Cela rend d'autant plus plausible ses malaises ressentis à l'estomac, partie du corps souvent privilégiée pour les manifestations du stress et de la nervosité exacerbée.

À ces modifications de la caractérisation des personnages s'ajoute un autre type de transformation qui, lui, est de l'ordre du discours. «Discours» est employé ici dans son sens tradition- 
nel. Ce type de transformation formera la deuxième catégorie des modifications qui touchent à la macrostructure du récit. Non seulement ce passage du discours écrit au discours oral affecte chacune des scènes du film, mais le dialogue court de l'une à l'autre, traverse l'œuvre tout entière, de la même manière que la caractérisation des personnages refait surface selon les nécessités du récit.

Ce qui sera dans la version cinématographique échange verbal entre personnages apparaît d'abord sous forme de dialogues écrits dans le récit scénaristique. Souvent ce type d'écriture tient compte de l'oralité future du dialogue et développe une technique qui veut la manifester le mieux possible. L'élision de certains phonèmes, ou même une construction syntaxique différente de la langue écrite, pour ne mentionner que celles-là, sont des possibilités offertes à l'écrivain pour suggérer l'oralité du texte. Nous sommes tous assez familiers avec ce genre d'écriture où les «il» deviennent des «y», les «je suis» se transforment en «j'suis» ou encore les «ça» remplacent les «cela».

Néanmoins ce type d'écriture ne peut pas manifester toutes les subtilités dont est capable l'oralité. Il ne peut que suggérer, faire comme si. La dynamique de l'oralité, et peut-être même la personnalité du comédien, si le réalisateur lui en laisse la possibilité, génèrent des modifications qui vont parfois au-delà de la structure du mot ou de la syntaxe. Il n'est pas dans notre intention d'aborder dans le détail ce genre de modification ${ }^{5}$. Soulignons au passage que même des expressions complètes peuvent se modifier sous la pression de la langue parlée. Pouvoir intime en présente un bel exemple. Découvrant Thomas qui a un haut-lecoeur et qui s'est réfugié aux toilettes des hommes, Jean-Baptiste (Martial dans le film) lui dit: «T'es blanc comme un lavabo, relaxe, j'vais prendre ta place pour ce midi» (p. 65). Dans le récit filmique ce dialogue devient: «T'es blanc comme un drap, j'va y aller.» L'expression «blanc comme un drap» nous apparaît plus près du langage populaire. Remarquons le «j'vais prendre ta place pour ce midi» qui se contracte encore davantage en «j'va y aller", ne conservant que l'essentiel du message pour la bonne compréhension de l'évolution de la situation.

De plus, nous ne pouvons passer sous silence le fait que des modifications au niveau du dialogue peuvent avoir des répercussions sur, entre autres, la motivation des personnages. Dans le scénario, pour convaincre Théo d'accepter l'affaire, Meurseault, le policier complice, s'adresse à lui en ces termes: «T'as pas un fils? Ça pourrait l'aider à s'en sortir lui...» (p. 5). Meurseault semble vouloir suggérer que l'argent qu'en retira Théo pourrait permettre à son fils de mener une vie meilleure. Dans le récit 
filmique la différence est marquante. Meurseault transforme la deuxième partie de l'énoncé par: «Y pourrait t'aider.» C'est toute la fonction, le rôle du jeune garçon qui est modifié par ce remaniement du dialogue. De simple motif, Robin (le fils de Théo) se voit assigner un rôle de participant.

Le troisième type de transformation que l'on retrouve au niveau de la macrostructure est de l'ordre de l'organisation «interséquentielle» du récit filmique. Ces transformations sont généralement prises en charge par le montage. En fait, il y a soit élimination, ajout ou déplacement de scènes. Cela peut parfois entraîner des modifications majeures dans la structure du récit et même porter atteinte à l'histoire.

Attardons-nous d'abord à l'élimination de scènes. On en dénombre seize qui, du scénario au film, ont disparu6. La majorité d'entre elles n'ont pas d'incidence majeure sur l'histoire. Tout comme il en sera pour le déplacement de scènes, la fonction de ces retraits nous semble être d'intervenir au niveau du rythme du film. Il s'agit vraisemblablement d'éliminer des informations plus ou moins superflues qui n'auraient pour effet que de ralentir la cadence des actions. Rythme dont la rapidité est d'autant plus importante qu'il s'agit d'un thriller.

Par contre, il est important de noter que l'élimination de l'une d'entre elles, non seulement chambarde complètement la structure narrative du récit, mais a un effet direct sur l'histoire ellemême. La première scène du scénario nous situe en fin d'aprèsmidi dans la maison de Roxanne. Janvier (on reconnaîtra plus tard dans le récit qu'il s'agit de lui) arrive dans la maison, pointe son arme sur la nuque de Roxanne qui est assise à une table. «Un sourire commence à se dessiner sur les lèvres de Roxanne, mais il disparaît aussitôt... » (p. 2). Ce sont les derniers mots de la scène 1. À la seconde scène, nous nous retrouvons au centre de détention au moment de la libération de Théo et de la proposition que lui fait Meurseault.

L'histoire relate des événements qui ont déjà eu lieu. La présence de Roxanne et de Janvier dans la première scène est la résultante de cette histoire dont la scène 2 nous présente les prémisses.

Il faut cependant préciser que ce glissement temporel entre la scène 1 et 2 n'est, dans le scénario, que très difficilement repérable. Les indices marquant l'antériorité de la seconde scène par rapport à la précédente sont pratiquement absents. La confirmation en scène 1 de la présence de Janvier aurait pu laisser sous-entendre ce retour en arrière de l'action, car la suite du récit nous fera assister à sa première rencontre avec Roxanne. 
Cependant rien dans la scène ne laisse présager que le personnage présent avec Roxanne est Janvier. De ce personnage qui fait irruption dans la pièce, nous ne connaîtrons que peu de chose si ce n'est que «la main qui tient le revolver paraît tendue, nerveuse» (p. 2). Ce ne sont que les dernières scènes qui permettront de véritablement interpréter le récit comme un retour en arrière. Conclusion dont nous n'aurons la certitude qu'à la scène 95 où, dans un «Plan d'ensemble: on reconnaît Janvier debout devant Roxanne» (p. 168). Sur la table on reconnaît également «deux objets: le revolver offert par Gildor, et le couteau offert par Jean-Baptiste» (p. 168). Double certitude même, puisque cette scène, qui confirme la présence de Janvier, s'affiche explicitement comme la continuité de la première scène du récit scénaristique: «En plan rapproché, un couteau vient se planter près d'un verre renversé (suite scène 1)» (p. 168).

Si le récit s'articule autour d'un retour en arrière, il est alors possible de considérer l'histoire qui nous est racontée comme la remémoration d'un souvenir. Et ce souvenir, il appartient de toute évidence à Roxanne. C'est le seul personnage de cette scène qui nous est véritablement présenté. On peut lire dans le scénario qu'«on peut voir en détail le visage du personnage... Un long profil d'androgyne... Roxanne, le regard fixe, se redresse lentement sur sa chaise» (p. 2). De plus, c'est par elle qu'est introduite la deuxième scène du récit scénaristique; scène qui, nous l'avons vu, se trouve être aussi la première du long retour en arrière qui durera jusqu'à la scène 95 , l'avant-dernière scène du film. Si nous n'en sommes pas convaincus à la fin de la première scène, tous nos doutes disparaissent lorsqu'à la scène 95 Roxanne elle-même nous le précise.

ROXANNE. - C'est drôle quand tu m'as touchée [elle fait le geste de la main] j'ai r'passé d'un coup tout ce qui vient de m'arriver...

Comme si t'avais pesé sur un bouton... (p. 168).

De cette manière de procéder, l'on peut dégager l'observation suivante: le récit accorde une importance appréciable au personnage de Roxanne. C'est à travers elle que l'histoire nous est racontée; tout au moins en est-elle l'élément déclencheur. Avec une semblable ouverture, nous sommes en droit de nous attendre à ce que ce personnage ait un rôle d'importance dans la suite du récit qui devrait nous relater son itinéraire, son cheminement, ses découvertes, ses hésitations... Il est cependant difficile d'affirmer que Roxanne est bien le pivot du récit, même si elle possède certains traits caractéristiques de la mythologie héroïque 7 . Entre autres choses, Roxanne, personnage androgyne, vient de l'extérieur, on ne sait trop d'où, pour remplir une mis- 
sion puis, une fois cette mission accomplie, repart vers un ailleurs inconnu.

Par contre, ce centrage momentané du récit sur Roxanne, ou du moins cette insistance sur le personnage, nous permet de mieux comprendre les dernières scènes qui recentrent le récit scénaristique sur elle. Toutefois, si la première scène trouve sa résonance dans les dernières, rien dans le développement du récit ne prépare ni même ne laisse entrevoir la finale: elle et Janvier partiront ensemble.

Le récit filmique procède tout autrement. Il élimine la première scène du scénario et nous plonge directement au coeur de l'action. Le récit est ici linéaire et chronologique. De cette manière Roxanne perd son rôle d'embrayeur du récit. Notons qu'elle y retrouve par contre une autre caractéristique du personnage type que nous décrivions plus haut. Au lieu de quitter la maison avec Janvier, ce qui constituerait un certain écart face à la mythologie héroïque, c'est seule qu'elle repartira vers un ailleurs tout aussi indéfini que celui d'où elle venait.

$\mathrm{Ce}$ décentrement du récit par rapport au personnage de Roxanne nous semble plus conforme à l'évolution dramatique, à la fois de l'action et des personnages. Aucun d'entre eux ne «porte» d'une façon prédominante le récit filmique; chacun à tour de rôle y tire son épingle du jeu. Le départ de Roxanne qui laisse derrière elle Janvier est plus conforme à la logique du récit où rien ne laisse présager une telle intimité entre les deux personnages.

Du récit filmique de Pouvoir intime se dégage un autre type de modification possible de son organisation interséquentielle: l'ajout de scènes. Dans le scénario, le personnage de Robin, le fils de Théo, est introduit par une suite de scènes (scènes 17-1819-20) dans lesquelles le jeune homme, après avoir ramassé quelques objets dans un appartement, rejoint son père à l'endroit où il se terre (c'est un délateur!) en ayant bien pris soin de semer deux individus qui le suivaient.

À l'écran, le personnage de Robin intervient dès le début du récit (scènes $3 \mathrm{~A}$ et $3 \mathrm{~B}$ selon notre découpage). Il est dans un bar où des clients s'amusent autour de quelques tables de billard. Deux individus entrent. Visiblement, ils recherchent quelqu'un. Robin comprend très vite qu'il peut s'agir de lui et quitte subrepticement les lieux. Par la suite, ce n'est plus à la cachette de Théo qu'il rencontrera son père, comme cela était prévu dans le scénario, mais dans un appartement qui est plausiblement celui de Robin. C'est là que le père et le fils décident de se cacher pour un certain temps dans un entrepôt désaffecté. 
Le générique d'ouverture du film peut lui aussi être considéré comme une scène ajoutée puisqu'il joue un certain rôle au niveau des actions. Alors que le générique déroule ses informations habituelles, on entend en son «off» deux voix masculines qui, par téléphone, se donnent rendez-vous. Nous reconnaîtrons plus tard les voix de Meurseault et de H.B., le policier et le fonctionnaire impliqués dans l'affaire. Dans le scénario, plus conventionnellement le générique vient se superposer à la rencontre de Meurseault et Théo au centre de détention (scène 2) pour se poursuivre pendant la scène suivante alors qu'on nous présente Roxanne qui se prépare à prendre un train.

Nous avons déjà fait allusion, plus haut, au passage de l'histoire où Thomas, pris d'un malaise, se réfugie aux toilettes des hommes alors que sa fonction est de garder le camion (scène 30 du récit scénaristique). L'entrée de Thomas dans le restaurant est intégrée dans le scénario à la scène 29 et relayée à l'arrière-plan: «Janvier porte sa facture à un client. En arrièreplan, perdu à travers toute l'activité du restaurant, on entrevoit Thomas qui entre et se dirige rapidement vers les toilettes» (p. 63).

On remarque dans ce passage une problématique intéressante de «transsémiotisation». Il est impossible à la lecture du scénario de manquer l'entrée de Thomas. Même si le texte précise qu'elle se fait à l'arrière-plan et comme dissimulée par l'activité qui règne au restaurant, il nous est impossible de ne pas le lire (à moins de sauter le passage). Transposé à l'écran, la lecture d'un tel plan, parce qu'il contient une foule d'informations, peut ne pas être complète. Il nous est alors possible de rater l'entrée de Thomas «perdu à travers toute l'activité du restaurant».

Moins allusif, le récit filmique réserve une scène spécifique à la sortie de Thomas. Ce départ se fait sous le regard surpris de Meurseault qui, de l'extérieur du restaurant, surveille le développement de la situation. Et c'est en gros plan que nous assistons à l'entrée de Thomas dans le restaurant. Le récit filmique repousse donc la proposition allusive du scénario pour préférer une voie plus explicite qui favorise davantage la construction d'un suspense. Plus question que l'on rate l'entrée de Thomas, elle se fait directement sous nos yeux. De plus, la réaction de surprise de Meurseault, sorte d'ancrage à l'interprétation de la situation, souligne son anormalité.

Il nous reste à parler du déplacement, dernier type de modification dans l'organisation des scènes. Ces déplacements ont pour effet soit de rendre plus rapidement accessible aux spectateurs(trices) une information, soit de la retarder. Comme unique exemple nous mentionnerons le déplacement d'une partie 
importante du dialogue entre H.B. et Meurseault de la scène 23 du scénario qui apparaît dans le récit filmique à la scène 2 . Il résulte de cette modification que le(la) spectateur(rice) de cinéma prend conscience beaucoup plus tôt dans le récit de l'environnement éminemment politique dans lequel baignera toute l'histoire.

\section{Modifications d'ordre microstructurel}

Dans cette catégorie de modification nous retrouverons en partie les mêmes types de transformation que ceux qui affectaient l'organisation interséquentielle du récit filmique. Une différence cependant les distingue. Les éliminations, ajouts ou déplacements modifieront dans le cas présent la structure à l'intérieur de la scène elle-même et ne porteront aucunement atteinte à l'intégrité de la structure globale du récit. À cela s'ajouteront des modifications qui affecteront la distribution du dialogue, tandis que nous regrouperons toute une autre série de modifications qui touchent à ce que nous nommerons la mise en scène ${ }^{8}$.

La courte scène 13 offre un bel exemple à la fois de coupure et d'ajout d'une partie de scène. Roxanne et Gildor sont au restaurant en face duquel aura lieu le vol. Il lui a fait un topo de la situation telle qu'elle devrait se présenter lorsque le jour sera venu. Elle quitte Gildor et se dirige vers le couloir des toilettes du restaurant.

Rassemblées en un tas grouillant et bruyant, une douzaine de touristes se racontent leur journée en attendant leur tour pour la toilette des femmes. Roxanne n'a pas le goût d'attendre, et c'est sans trop se faire remarquer qu'elle pousse la porte de la toilette des hommes. Une des touristes regarde à deux fois vers la porte de la toilette des hommes qui se referme, comme pour s'assurer qu'elle a bien choisi, elle, la bonne porte (p. 25).

Dans le récit filmique, disparaît le «tas grouillant et bruyant» de la douzaine de touristes; il se réduit à deux femmes. Et ce n'est plus de leur journée qu'elles discutent. L'échange est amorcée par l'arrivée de Roxanne.

TOURISTE 1, à Roxanne. - Y'a du monde hein!

ROXANNE. - Ouais!

[Elle quitte le cadre.]

Disparaît également le jeu de la touriste qui s'assure de la porte qu'elle a choisie. Roxanne, elle, ne fait rien pour dissimu- 
ler l'endroit où elle se rend. D'ailleurs de cette modification découle l'ajout d'un autre dialogue.

TOURISTE 2. - Aye! A rentre dans la toilette des hommes!

TOURISTE 1. — Hein!

[Scandalisées, les deux se rẹgardent...]

À la scène 24 du scénario, Théo et son fils Robin arrivent à l'entrepôt du théâtre, là où se trouve le quartier général de leur opération. Roxanne apprend que Robin fera partie de l'équipe, elle «reste étonnée» (p. 49). Théo lui annonce, ainsi qu'à Gildor, que «c'est demain que ça se passe!» (p. 50).

À cet endroit, le récit filmique modifie l'organisation interséquentielle du scénario en arrêtant le développement de cette scène pour faire place à une discussion entre Meurseault et H.B. Dans le récit scénaristique cette discussion a lieu au cours de la deuxième partie de la scène 23 . Une fois la confrontation entre H.B. et Meurseault terminée, le récit filmique nous ramène à la situation de la scène 24 du scénario.

Toutefois, ce retour ne se fait pas sans coupures. Trois pages du scénario ont disparu. L'action reprend alors que Théo décide de faire une répétition. Ainsi le récit filmique gomme l'interrogation de Roxanne sur la présence de Robin. Est éliminée également une série d'actions montrant l'équipe au travail. Partie de scènes dont la fonction est de rapprocher Robin et Roxanne, de faire en sorte que l'adolescent devienne un membre accepté de tous en prouvant son utilité.

La scène 79 présente elle aussi des modifications importantes où tout un passage du récit scénaristique est condensé en une seule image. Enfermé dans le camion, Jean-Baptiste (Martial dans le film) «ressemble de plus en plus à un soldat durant un état de siège. Il a perdu la notion du temps. (...) Jean-Baptiste recrée son univers et parle tout bas, pour lui-même» (p. 141). Dans le scénario cet étrange voyage intérieur qui se fait par l'intermédiaire des photos que lui a remises Janvier couvre environ une page et demie. Le récit filmique condense tout cela en une seule image subjective et très expressive où, à travers les yeux de Martial (Jean-Baptiste dans le scénario), on découvre l'intérieur arrière de la boîte du camion démesurément allongée. À cet instant, cette partie du camion ressemble à un interminable corridor.

On peut également relever certaines modifications dans l'ordre des actions ou des gestes à l'intérieur d'une scène. C'est la première rencontre, le terme confrontation serait peut-être 
plus juste, entre Roxanne et Théo. Ce qu'il voit ne l'emballe guère.

ROXANNE. - Cherchiez-vous quelqu'un de plus féminin...?

THÉO. - Humm... oui... Genre secrétaire peut-être.

[Gildor s'empresse...]

GILDOR. - Ha! mais ça ça s'arrange, c'est juste une question de vêtements... de costumes! Tu peux avoir l'air d'une secrétaire.

ROXANNE. - Je sais pas.

[Sourire de Roxanne, suivi de ceux de Théo et de Gildor. La complicité s'installe] (p. 16) ${ }^{9}$.

Le scénario nous présente donc Roxanne comme l'initiatrice de cette complicité; tout au moins est-ce elle qui, par son sourire, sollicite l'adhésion de Théo. Par la suite, le sourire de Gildor marque son contentement par rapport à la position prise par Théo, le «patron», le responsable de toute l'affaire.

Le processus s'inverse dans le récit filmique et semble suivre un trajet plus conforme à l'attitude générale du personnage de Roxanne: attitude distanciée, du moins distante et réservée. Suite au «je sais pas» de Roxanne, Théo fait durer le suspense au cours d'une pause où il examine la femme. Sa réaction est plus qu'importante. Les deux autres attendent le verdict. Alors Théo sourit, confirmant ainsi l'hypothèse de Gildor. D'ailleurs Théo acquiesce en portant son regard sur ce dernier qui, heureux du verdict (on peut supposer que c'est lui qui avait proposé Roxanne comme équipière), sourit à son tour. Ce n'est qu'à ce moment que Roxanne, maintenant rassurée, peut sourire. Et encore! Le film, qui passe rapidement à la scène suivante par une coupe franche, ne nous présente qu'une amorce de sourire. Par contre Roxanne manifeste une satisfaction évidente qui n'efface cependant pas la réserve qu'elle pose entre elle et les autres depuis le début du récit

Nous retrouvons une autre différence majeure dans l'organisation interne des scènes lorsque l'on compare la fin de chacun des récits. Dans le scénario, Roxanne et Janvier découvrent qu'ils partagent des points de ressemblance; surtout Janvier qui décide de partir avec elle. Le film élimine tout ce rapprochement entre les deux personnages, et c'est seule que Roxanne quitte la maison.

Nous avons regroupé d'autres modifications d'ordre intra-séquentiel autour du terme générique de «mise en scène». Nous y 
retrouverons des modifications relatives à la mise en place, au choix des décors, et à la mise en cadre. Nous y avons très arbitrairement inclus les indications d'éclairage et les effets sonores.

Par rapport au récit de Simoneau et Curzi, le récit filmique manifeste certaines modifications relatives aux décors, plus précisément à ce qu'on appelle en termes du métier: les locations. Ces changements n'altèrent pas la structure du récit mais modifient le lieu de la scène.

Contentons-nous de ne mentionner qu'un exemple pour illustrer ce type de transformation. Dans le film, la première rencontre de Meurseault et H.B. se fera dans une pièce qui semble située au sous-sol d'un édifice car, avec H.B., y sont deux hommes affairés à réparer ce qui ressemble à une fournaise ou quelque chose du genre. Pour accéder à cet endroit, Meurseault traverse comme un long corridor très sombre, et c'est à contrejour, face à une grande fenêtre très éclairée, que se fera la discussion entre les deux hommes. Le scénario situe cette rencontre au «Q.G. de Meurseault», un appartement qui surplombe la ville.

Ce changement de location peut peut-être s'interpréter à la lumière du fait que dans le récit filmique ce tête-à-tête se produit en scène d'ouverture. La machine n'est pas encore véritablement mise en marche; la mission non encore acceptée. Présenter les instigateurs du vol dans un lieu plus sombre que l'appartement à l'atmosphère somme toute moins lourde du «Q.G. officiel», souligne avec plus d'acuité l'aspect «underground», confidentiel, du travail qui est confié à Meurseault. Et comme nous le soulignions, la mission n'est pas encore véritablement effective. Ce n'est que lorsque le train sera lancé sur ses rails que nous retrouverons ensemble les deux personnages, et au «Q.G.» cette fois, comme il se doit.

Sur le chemin du retour, alors que Gildor est allé chercher Roxanne à la gare, les deux discutent tandis que l'automobile, dans laquelle ils ont pris place, roule. Laissons les scénaristes présenter la situation:

INTÉRIEUR. JOUR. VOITURE

\section{- VOITURE DE GILDOR}

La caméra cadre le rétroviseur de la voiture; un petit palmier qui s'y trouve accroché se balance de gauche à droite tandis qu'en arrière-plan, le paysage qui défile à vive allure reste flou.

Échange rapide (p. 11).

Cet échange entre Roxanne et Gildor porte sur le comment de leur nouvelle rencontre et surtout marque le fait qu'ils se 
connaissent depuis un certain temps. Le petit palmier qui doit couvrir tout (ou presque) l'espace visuel occupe donc une place centrale puisque la discussion qui suit se fait en son «off» laissant toute la présence écranique à ce «souvenir» (p. 11). Et pourtant, rien dans le récit scénaristique ne vient d'une manière ou d'une autre justifier cette prédominance de l'objet. Ce souvenir de voyage n'unit en rien Roxanne et Gildor, il n'a rien à voir avec leur relation passée. Malgré cela, le récit s'efforce d'établir un lien étroit entre cet objet et Roxanne. C'est la «main de Roxanne (qui) entre dans le cadre et immobilise le petit palmier accroché au rétroviseur» (p. 11). C'est encore elle qui questionne l'origine du palmier: «Un souvenir de voyage...?» (p. 11) Et comme si ce n'était pas encore assez, le texte du scénario se poursuit en précisant, après une réplique de Gildor, qu'apparaît le «visage de Roxanne (qui) remplit la $1 / 2$ de l'écran à son tour: l'image du petit palmier qu'elle tient maintenant entre ses doigts se réfléchit dans ses verres fumés» (p. 12). Précisons que dans le film Roxanne ne porte pas de verres fumés.

Cette insistance visuelle dans le scénario sur le petit palmier passe tout à fait au second plan dans la version filmique. L'objet lui-même se transforme et devient un trousseau de clefs dont, d'ailleurs, on ne sait trop si Gildor, de la manière dont il le tient, montre à Roxanne ou le trousseau de clefs lui-même ou bien uniquement les clefs. La scène se présente comme si l'importance visuelle que le scénario accordait à l'objet passait au second plan ${ }^{10}$ pour centrer maintenant le récit sur le dialogue. Par contre, le récit filmique, lui, fait du trousseau de clefs un point de convergence, un symbole pourrait-on dire, de leur passé partagé. Cette fois c'est Gildor qui devient le personnage associé à l'objet; c'est lui qui tient dans sa main le trousseau de clefs. Signalons de plus un changement de location. Dans le récit filmique cette partie de l'histoire se déroule non pas dans l'automobile mais à la gare de chemin de fer alors qu'ils traversent un long corridor.

GILDOR. - Te rappelles-tu?

ROXANNE. - Non. Un souvenir de voyage?

GILDOR. - Tu t'en souviens pas?

ROXANNE. - Non... Comment ça va se passer?

Est-ce parce qu'il est un peu offusqué de la mémoire oublieuse de Roxanne qu'il ralentit le pas après la réponse brève mais assurée de cette dernière? Elle qui, sans même porter attention à la 
déception de Gildor, oriente la conversation vers une tout autre direction en enchaînant presque immédiatement sur le comment du déroulement de l'action à venir. Est-ce pour la même raison que c'est en pressant le pas pour la devancer qu'il répond à son interrogation? Nous sommes en droit de le croire.

Étrange renversement de situation que nous présente cette scène. D'un texte écrit mais qui met indéniablement l'accent sur l'aspect visuel de la situation, le film, intrinsèquement iconographique, «gomme» d'une certaine manière cette réalité pour donner préséance au discours oral. Et pourtant ni l'un ni l'autre des récits ne parviennent à créer un lien structurel fortement cohérent entre la situation et sa manière de la présenter, ou dit plus banalement et plus traditionnellement, entre le fond et la forme. Le récit scénaristique suggère la présence très forte d'une image qui aurait pu servir de lieu de convergence, de lieu de rencontre du passé commun des protagonistes. Au lieu de cela le récit prend une tout autre direction. La relation triangulaire que le petit palmier aurait pu instaurer (Roxanne / palmier / Gildor) s'évanouit pour n'apparaître que comme prétexte à l'effet visuel. Le récit filmique ne parvient pas lui non plus à établir cette cohérence. Cette fois l'objet du souvenir sert bien de pont entre les deux personnages. Roxanne n'en a plus souvenance, soit, mais le trousseau de clefs nous est bien présenté par Gildor comme étant, non plus comme dans le scénario un simple souvenir dont on ne sait trop à quoi il est rattaché, mais bel et bien comme une preuve d'un passé partagé. Cependant, si le texte nous suggère cette orientation du récit, l'image, celle de l'objet souvenir, n'a plus la force «visuelle» que lui prêtait le récit scénaristique. De prédominante, elle se confond à l'intérieur d'une image, d'un cadre beaucoup plus large et dont, par le fait même, la somme des informations accessibles aux spectateurs(rices) est grandement augmentée. D'image carrefour, comme elle était suggérée dans le scénario (même si nous savons que ce dernier ne parvient pas à lui faire jouer ce rôle), le récit filmique la dilate pour la ramener à une dimension moins lourdement signifiante visuellement et ainsi perdre une bonne partie de sa force d'impact et de son originalité.

La scène 40 offre un bel exemple de changement de ce que nous nommons la mise en place. On vient rapidement de quitter les lieux de la fusillade. Roxanne est dans la voiture de Gildor qui se concentre sur la conduite.

Roxanne ouvre la boîte à gants, prend une cigarette dans un paquet, s'allume et referme d'un coup de poing le couvercle de la boîte à gants. Elle regarde dehors (p. 76). 
Dans le récit filmique, Roxanne est assise à l'arrière de l'auto. Au moment où la scène débute, elle enjambe l'espace étroit qui sépare les deux banquettes avant et s'assoit du côté du passager. Une fois installée, elle arrache la perruque qu'elle porte en s'exclamant: «Maudit!» Puis on passe immédiatement à un plan de Martial (Jean-Baptiste dans le scénario) allongé dans la boîte arrière du camion alors que Théo et Robin sont installés à l'avant. Rapide, dynamique, le récit filmique évite le possible piège du ralentissement de l'action que peut entraîner une cigarette que l'on allume.

Si nous avons précédemment insisté sur la scène du souvenir initiée par le petit palmier (ou le trousseau de clefs dans le film), c'est aussi parce que ce thème est récurrent dans l'œuvre. Il ressurgit alors que, parce qu'il veut instruire la nouvelle partenaire des habitudes des gardiens, Gildor s'est rendu avec Roxanne au restaurant en face duquel aura lieu le vol du camion. Un début de conversation explique comment Gildor a connu Théo:

Les souvenirs courent dans la tête de Gildor. Il détaille minutieusement le visage de Roxanne, puis rompt le silence.

GILDOR. - Ça te change beaucoup, les cheveux courts... t'as l'air plus sérieuse...

Roxanne, sourit, Gildor aussi (p. 22-23).

Le récit filmique minimise cette résurgence du souvenir. À ce moment l'image nous montre, assis au comptoir, Gildor cadré en amorce de dos à gauche de l'écran alors que l'on voit en gros plan le visage de Roxanne ${ }^{11}$. Un tel cadrage rend impossible pour le (la) spectateur(rice) de vérifier de visu si Gildor «détaille minutieusement le visage de Roxanne». Et loin de rompre le silence (ce qui, venant suppléer le visuel, aurait pu à tout le moins donner l'impression que Gildor s'était attardé longuement sur le visage de Roxanne), il reprend la parole après une pause de courte durée. De plus, l'attitude de Roxanne pendant cette brève pause ne donne aucunement l'impression qu'elle subit ou qu'elle est troublée par le regard de Gildor. Cela a pour effet d'annihiler complètement l'impact d'un silence rompu. Aucun temps suspendu n'est ménagé au souvenir pour qu'il puisse trouver sa place et courir «dans la tête de Gildor». Il ne poindra qu'à travers sa réflexion à propos du changement de coiffure de Roxanne.

Lancé sur la piste du souvenir, le scénario tente, dans la même scène, d'y attirer Roxanne. La remarque de Gildor sur la nou- 
velle coiffure de sa partenaire est immédiatement suivie d'un jeu visuel devant éveiller chez elle un souvenir précis.

GILDOR. - Tu te souviens de ça?

[Dans une série de gestes silencieux, Gildor mime un souvenir dans l'espace. Son attitude caricaturale, presque clownesque, surprend Roxanne. Elle échappe un rire qui stimule Gildor un peu plus; Il (sic) rit à son tour. Il essaie de poser sa main sur celle de Roxanne qui, dans le même mouvement, retire la sienne. Petit temps froid et vide.]

[Un garçon de table (Janvier) vient porter l'addition. Roxanne se lève comme pour quitter le restaurant] (p. 23).

Le jeu visuel suggéré par le scénario est remplacé dans le film par un jeu «oral». Abandonnant la piste de l'éveil d'un autre souvenir chez Roxanne, le récit filmique reste centré sur celui suggéré par la chevelure.

GILDOR. - Ça te change beaucoup, les cheveux courts... J'aime ça... T'as l'air plus sérieuse...

ROXANNE, léger rire. - Oui...

GILDOR. - T'avais tellement de cheveux avant. La première fois que je t'ai vue c'est ça qui m'a frappé: ta crinière.

ROXANNE. - Humm... Humm...

GILDOR. - C'est beau comme ça aussi.

ROXANNE. - Ouais...

GILDOR. - C'est différent.

ROXANNE. - Ah! ouais... ouais...

GILDOR. - Non, non, j'aime ça c'est vrai.

ROXANNE, rit. - Viens on bouge.

Aucun besoin dans le récit filmique du secours du garçon de table pour venir combler un «vide» maintenant inexistant. Jamais Roxanne, dans cette scène, n'est amenée à avoir un contact qui génère entre elle et Gildor un petit «temps froid et vide». La distance entre les deux personnages y est constamment maintenue malgré la présence d'une certaine complicité. De toute évidence, le scénario accorde à ces deux personnages l'existence de liens sentimentaux; le film semble non pas tant s'y refuser que d'en éviter une émergence trop forte. Respectant en cela les indications du scénario, le ton reste au niveau de la 
blague, de la taquinerie. Jamais il ne se teintera du «sérieux» que génère le geste de Gildor dans le récit scénaristique. Roxanne peut alors elle-même prendre en charge la relance du récit sans le secours, tout prétexte qu'il soit, du garçon de table. Notons également qu'une fois encore une description «visuelle» du scénario est relayée par l'oralité dans la diégèse du film.

Certaines indications sonores subissent elles aussi des modifications lorsqu'elles apparaissent dans le récit filmique. Lorsque Meurseault téléphone à Thomas pour lui confirmer que le vol se produira le midi même (scène 26), il est indiqué dans le scénario que d'une «radio s'échappe une musique aux accents western (sic)» (p. 59). Il est permis de croire que cette musique caractérise le personnage de Thomas. Le récit filmique, quant à lui, fait fi de ce trait distinctif. Aucune musique, qu'elle vienne d'une radio ou non, n'est présente dans cette scène. En fait, le silence n'est brisé que par la profonde toux de Thomas et la sonnerie du téléphone. Cependant, justement parce qu'ils ponctuent le silence de la scène en occupant tout l'espace sonore, ces deux sons acquièrent une place prépondérante dans le récit. Et pour cause! Ce sont évidemment deux éléments importants dans cette histoire que la toux de Thomas et le coup de téléphone de Meurseault. Le premier dénote la fragile santé de Thomas qui sera le pivot de l'échec de l'entreprise; l'autre confirme l'imminence du vol.

Remplaçant Thomas dans le camion blindé, Jean-Baptiste (Martial dans le film) regarde les photos que lui a remises Janvier. «Le bruit extérieur, déjà étouffé par le blindage du camion, est à peine perceptible à travers la musique qui s'échappe d'un radio portatif (sic)». La scène se termine alors que la «musique nasillarde et agressive du poste de radio remplit la bande sonore» (p. 68).

Dans le récit filmique, c'est dès le début de la scène que la musique remplit la totalité de l'espace sonore. Le bruit extérieur est moins qu'«à peine perceptible», il est inexistant. De plus, cette musique ne provient pas d'une radio portative qui se trouverait à l'intérieur de la cabine du camion. Bien avant cette scène, elle a déjà commencé à envahir la bande sonore. Elle débute avec l'arrivée du camion blindé en face du restaurant pour se terminer lors de la prise de Roxanne en otage. Si elle se retire pendant un certain temps à l'arrière-plan sonore, elle évacue toute autre présence auditive dès la sortie de Martial (JeanBaptiste dans le scénario) du restaurant pour aller remplacer Thomas. Et à aucun moment à travers ce long segment du récit, la présence d'une radio portative ne vient ancrer dans l'image la 
provenance de cette musique. Il s'agit bel et bien d'une musique extra-diégétique. Aucunement «nasillarde et agressive», elle marque la construction d'une tension dramatique.

Par cette modification, le rôle de la musique n'est plus de singulariser la scène des photos, souvenirs de vacances passées avec Janvier, en accentuant le contraste entre Jean-Baptiste (Martial dans le film) qui «se laisse glisser dans un autre univers plus heureux» (p. 68) et l'agressivité de la musique, sorte d'anticipation sonore sur le développement futur du récit. Sa fonction dans l'œuvre à l'écran est plutôt d'homogénéiser ce segment du récit pour mettre tous les éléments au service d'une même cause: une lente mais inéluctable progression dans le développement des situations qui mettent en place le tragique dérapage, avant-goût de l'hécatombe finale.

Plusieurs indications de cadrage sont également présentes dans le scénario de Pouvoir intime. Les modifications qu'elles subissent nous semblent généralement moins significatives que certaines autres mentionnées précédemment. L'une d'elles, relativement plus importante, survient à la scène 66. Meurseault et H.B. sont à leur quartier général. Théo vient d'appeler pour qu'on lui envoie un médecin car son fils a été grièvement blessé par Jean-Baptiste (Martial dans le film). Alors que les deux discutent, «H.B. fait dos à la caméra tandis que Meurseault marche de long en large en entrant et sortant du cadre» (p. 112). Ce manège se déroule pendant un certain temps. Puis, de retour dans le cadre, Meurseault

Relève ses manches, desserre sa cravate, sort du cadre

Lent traveling avant sur H.B. (...) Meurseault revient dans le cadre et s'arrête près de l'autre toujours de dos. L'attitude distante de H.B. exaspère Meurseault. (...) Fin du travelling: les deux sont maintenant plein cadre. (...) L'image de H.B., tirée à quatre épingles, contraste violemment avec la tenue débraillée de Meurseault. Ce dernier le regarde, puis sort du cadre (p. 112113).

Jouant sur ce contraste entre les deux hommes, le récit filmique tente de visuellement les isoler plutôt que de les rapprocher. Lors du lent travelling sur H.B., Meurseault revient effectivement dans le cadre mais c'est pour en ressortir presque aussitôt. Ainsi à la fin du travelling, ce ne sont pas les deux qui se retrouvent dans le cadre mais H.B., tout seul, qui s'est retourné face à la caméra. Ils resteront isolés, chacun dans leur cadre, jusqu'à la fin toute prochaine de la scène. Unis par la nécessité, un monde les sépare... 
À la scène 70 , pour forcer Martial à sortir du camion on introduit par un orifice un boyau d'arrosage qui déverse son eau à l'intérieur du blindé.

Jean-Baptiste essaie de ramasser ses idées durant une seconde... Un mince filet de lumière tombe alors sur son visage. La caméra pivote et vient cadrer le hublot de communication: quelqu'un est en train d'enlever le carton qui l'obstrue. Le visage de Roxanne apparaît, puis, partiellement, celui de Théo. (...) Dans un mouvement arrière la caméra nous laisse découvrir la tête de JeanBaptiste collé au mur où se trouve le hublot. Dans cette position ils ne peuvent le voir. Il retient sa respiration (p. 118-119).

Les scénaristes ont ici mis l'accent sur le suspense. Qui regarde? Nous ne le savons que lorsque la caméra a terminé de pivoter de Jean-Baptiste (Martial dans le film) à Roxanne. Où est Jean-Baptiste (Martial dans le film)? Même procédé. Nous ne le saurons que lorsque la caméra aura terminé son mouvement arrière.

Ce rôle de tenseur dramatique joué par la caméra (ou les mots qui en tiennent lieu!) est abandonné dans le récit filmique. La scène de l'introduction du boyau d'arrosage est cadré en plan américain et la caméra est à l'intérieur de la boîte arrière du camion face au hublot de communication. Ainsi, alors que JeanBaptiste (Martial dans le film) s'affaire à repousser le boyau, nous voyons en même temps quelqu'un qui enlève le carton obstruant le hublot. Ce geste à peine amorcé, on coupe rapidement pour nous montrer qu'il est accompli par Roxanne. L'absence de suspension momentanée de la progression de l'action, suspension dont la fonction est justement d'indiquer qu'il se prépare quelque chose, désamorce le suspense que laissait entrevoir le récit scénaristique ${ }^{12}$.

Il en va de même de la découverte de Jean-Baptiste (Martial dans le film) qui se cache pour ne pas être vu. Dans le récit filmique, après l'interrogation de Roxanne qui ne voit personne à l'intérieur de la boîte arrière du camion, c'est le même cadrage de Martial (Jean-Baptiste dans le scénario) en plan américain qui revient. Le(la) spectateur(rice) est donc immédiatement informé(e) de sa position. Si Roxanne a des doutes, le(la) spectateur(rice) n'en a aucun.

Il nous reste à mentionner comme type de modification relatif aux indications de cadrage: les mentions de son «off». Dans le scénario de Simoneau et Curzi, elles sont généralement associées au dialogue. Si nous n'avons pas classé ce type de modification dans la même catégorie des sons que ceux que nous avons précédemment étudiés, c'est à cause de la contiguïté que leur assigne 
le scénario avec le cadre. Préciser que ces sons sont «off», c'est déjà déterminer une certaine composition du cadre; c'est au moins en exclure la source du son «off». L'inverse est également juste. Un dialogue ou une réplique non «off» présuppose la présence à l'écran du ou des personnages qui parlent.

Nous avons déjà signalé certaines modifications apportées à la scène 8 , la scène du palmier. L'importance visuelle accordée au palmier poussait hors cadre une partie de la conversation entre Roxanne et Gildor.

RoXANNE, off. - Tu m'as pas dit... Comment t'as fait pour me retrouver?

GILDOR, off. - Un hasard...

ROXANNE, off. - Je te crois pas...

GILDOR, off. - Une série de hasards...

ROXANNE, off. - C'est déjà mieux...

[La main de Roxanne entre dans le cadre et immobilise le petit palmier accroché au rétroviseur.]

ROXANNE, off. - Un souvenir de voyage...? (p. 11)

L'on sait les modifications apportées par la mise en place, le changement de location et la résultante de ce passage du «off» au «in» dans le récit filmique.

La scène 75 nous présente la situation inverse: le passage du «in» au «off». Nous sommes au quartier général de Meurseault; lui et H.B. attendent les développements de la situation. H.B., dans la salle de bains s'apprête à renifler de la coke; Meurseault mange du poulet.

[Il jette un cil sur le poulet non entamé de H.B.]

MeURSEAUlT. - Vous mangez pas?

H.B. - Non, non, vous pouvez prendre ma part...

[Il nettoie l'évier méticuleusement, geste clinique] (p. 130).

En repoussant en hors cadre l'intervention accessoire de Meurseault, le récit filmique fait porter l'accent sur H.B. dont il est difficile de savoir, à ce stade, ce qu'il s'apprête vraiment à faire dans la salle de bains. L'insistance sur cette action permet de créer une certaine attente chez le(la) spectateur(rice) qui, possiblement, se questionne sur les gestes de H.B. L'intérêt dramatique est de cette façon maintenu plus efficacement que ne l'aurait fait un retour sur Meurseault qui s'interroge sur la faim du fonctionnaire. 
Une modification du même type est présente dans la scène, mais elle affecte cette fois l'autre personnage. H.B., dans la salle de bains, referme la porte derrière lui pour s'assurer qu'on ne peut le voir. Meurseault en profite pour inspecter les effets personnels de H.B. Afin de détourner l'attention de ce dernier, il alimente la conversation.

MEURSEAULT. - Savez-vous pourquoi les douanes
m'intéressent? [Dans la salle de bains, H.B. se repenche à nouveau hors cadre.]

H.B. - Vous voulez en apprendre davantage sur l'importexport?

[Il renifle.]

[De l'autre côté, la réplique fait rire Meurseault. Il réussit au même moment à ouvrir la mallette verrouillée] (p. 131).

Il n'est pas indiqué que la réplique de H.B. est en «off»; cela suppose que le récit, pour un instant, délaisse l'action accomplie par Meurseault pour se focaliser sur celle de l'autre personnage. Le récit scénaristique propose un fonctionnement en montage alterné: Meurseault / H.B. / Meurseault. Cette stratégie narrative n'est cependant pas mise en pratique dans le récit filmique qui focalise l'attention sur la fouille qu'exécute Meurseault. Si le récit filmique a tantôt retardé l'explication des gestes de H.B. dans la salle de bains, il prend soin de répondre à cette interrogation avant d'enclencher la phase de la fouille. À peine Meurseault a-t-il amorcé son inspection que le récit filmique nous conduit dans la salle de bains où on voit très bien, et non «hors cadre» comme l'indique le scénario, H.B. renifler sa drogue. Ce plan, en transmettant l'information nécessaire pour la bonne compréhension de cette partie du récit, comble les attentes du (de la) spectateur(trice) et permet par la suite de centrer toute l'attention sur Meurseault. En effet, c'est au hors champ et à la trame sonore qu'est confié le soin de supporter le discours de H.B. alors que Meurseault conserve tout l'espace visuel. Une autre fois le récit filmique repousse à l'extérieur du cadre une information accessoire, préférant mettre l'accent sur le suspense par le développement d'une situation à l'issue incertaine.

La scène 70 où Jean-Baptiste (Martial dans le film), caché dans la boîte arrière du camion blindé, tente de se soustraire au regard de Roxanne, nous permet de signaler un autre type de modification possible lors du transcodage d'un récit à l'autre: il 
s'agit d'indications d'éclairage. Le «mince filet de lumière [qui] tombe alors sur son visage [celui de Jean-Baptiste]» (p. 118) est inexistant dans le récit filmique. Aucun changement d'éclairage n'est perceptible lorsque Roxanne retire le carton du hublot.

Un dernier type de modification est encore repérable dans Pouvoir intime: une permutation dans la distribution des dialogues. Une modification de ce genre peut avoir des conséquences assez graves. Il n'en est rien dans le cas présent. Le changement de locuteur (scène 91) porte sur un énoncé exclamatif de surprise qui dit par l'un ou par l'autre des personnages ne change ni le sens, ni l'orientation du récit.

[Meurseault et H.B. regardent incrédules le camion qui vient dans leur direction.]

H.B. - Y se sauvent avec le camion!

MeURSEAUlT, en criant. - Y se sauvent pas, y nous foncent dessus!

Dans le récit filmique c'est Meurseault qui constate le départ du camion et c'est H.B. qui en précise la direction.

Les modifications que subit le récit scénaristique de Pouvoir intime lors de sa «transsémiotisation» permettent de le considérer comme une étape, un jałon dans le long processus de réalisation du film. Son véhicule sémiotique diffère totalement de celui du récit filmique. C'est pourquoi il est dès l'origine voué à la mutation. Parce que scriptural, le récit scénaristique ne peut qu'évoquer le réel, jamais le convoquer.

Cette métamorphose du véhicule de la transmission du sens entraîne dans sa foulée des modifications qui affectent la structure dramatique du récit. L'instantanéité et la polyphonie du récit filmique rendent possible une dynamique narrative que l'écriture, contrainte à la monodie et la consécution, ne peut réaliser.

Il s'ensuit que le type d'écriture du récit scénaristique peut être porteur d'écarts par rapport au récit filmique. Il existe une certaine codification de la présentation formelle d'un scénario. Cependant la manière de narrer les actions et de les mettre en contexte varie considérablement. Une écriture qui, tout en faisant porter l'accent sur l'action, accorde une place importante aux «descriptifs» (Viswanathan, p. 72) ou encore une autre où le développement des situations repose presque exclusivement sur le dialogue présentent certainement des écarts qui diffèrent de ceux relevés à partir des récits de Pouvoir intime.

À ces variables s'ajoute l'attitude du réalisateur. Un réalisateur qui prétend avoir un profond respect pour le scénario et un 
autre pour qui le récit scénaristique n'est qu'un brouillon de l'œuvre en gestation ne peuvent avoir la même attitude au moment du transcodage de ce récit. Les modifications apparaîtront possiblement plus nombreuses chez l'un, plus rares chez l'autre.

Ainsi ces autres paramètres permettraient de raffiner davantage le modèle typologique proposé. Son mérite actuel est de souligner l'importante étape dans la réalisation d'une œuvre cinématographique qu'est le passage parfois heureux, parfois moins, du scénario au film.

\section{Université Laval}

\section{NOTES}

1 Edward Chodorov, Positif, no 108, cité par Gilbert Maggi et France B. Maggi dans Langage et analyse filmiques 530-900, Lexique-Cinéma, 1976, p. 69.

2 L'idée originale, toutefois, est d'Yves Simoneau.

3 Bien que nous préférions le terme de «séquence», nous utiliserons celui de «scène» puisque c'est ce terme que nous retrouvons dans le scénario de Simoneau et Curzi.

4 Bien que dans le scénario ces indications apparaissent dans une rubrique appelée Description des personnages et qui précède le récit, nous les considérerons comme faisant implicitement partie de ce dernier. Précisons que ce mode de présentation des personnages répond aux exigences des institutions de notre cinéma. Il en va autrement ailleurs où ces indications peuvent apparaître dans le développement du récit lui-même.

5 Lire à ce sujet l'article de Michel Marie et Francis Vanoye, «Comment parler la bouche pleine», in "Énonciation et cinéma», Communications 38 (Paris: Seuil, 1983) p. 51-77.

6 Le scénario contient 96 scènes.

7 Voir à ce sujet Philippe Sellier, Le Mythe du héros ou le désir d'être dieu. Paris-Montréal: Bordas, 1970.

8 Nous donnons à ce terme la définition qu'en faisait Michel Deville lors d'un entretien télévisé. Selon lui son travail consistait à définir un cadre, un décor, une lumière. Nous ajoutons une mise en place, des indications d'interprétation, et pourquoi pas le son. Il s'agira pour nous, dans un travail subséquent, de préciser davantage cette classification.

9 La partie en gras réfère au film.

10 La caméra cadre ensemble Roxanne et Gildor en plan rapproché (taille) et en contre-plongée, ce qui a pour résultat de ne plus donner la prédominance visuelle à l'objet tenu par Gildor, c'est-à-dire le trousseau de clefs. 
11 Notons une modification relative à la mise en place: «Un peu à l'écart, dans un vaste restaurant, Gildor et Roxanne sont assis l'un en face de l'autre» (p. 22).

12 Notons l'absence de Théo dans ce cadrage. Il n'est pas près de Roxanne, mais à l'extérieur du compartiment avant du camion dont la porte est ouverte et où a pris place Roxanne.

\section{OUVRAGES CITÉS}

Curzi, Pierre et Simoneau, Yves, Pouvoir intime, le troc. Scénario non publié du film.

Jacqueline Viswanathan, «Une histoire racontée en images», Études françaises, vol. 22, no 3 (hiver 1987) p. 72-81. 